REVISTA EDUCACIÓN LAS AMÉRICAS

Facultad de EdUCACIÓN UNIVERSIDAD DE LAS AMÉRICAS
Revista Educación las Américas ISSN: 0719-7128

ccalisto@udla.cl

Universidad de Las Américas

Chile

\title{
El modelo pedagógico Flipped Classroom: contribución al desarrollo de aprendizajes y habilidades para la sociedad del siglo XXI
}

Espinoza Guzmán, Alejandro; Garrido Parraguez, María Paula; Martínez Villacura, Cristian

El modelo pedagógico Flipped Classroom: contribución al desarrollo de aprendizajes y habilidades para la sociedad del siglo XXI

Revista Educación las Américas, vol. 8, 2019

Universidad de Las Américas, Chile

DOI: https://doi.org/10.35811/rea.v8i0.3

Esta obra está bajo una Licencia Creative Commons Atribución-SinDerivar 4.0 Internacional. 


\title{
El modelo pedagógico Flipped Classroom: contribución al desarrollo de aprendizajes y habilidades para la sociedad del siglo XXI
}

\author{
Alejandro Espinoza Guzmán \\ Universidad Católica del Maule, Chile \\ aespinoza@ucm.cl \\ iD http://orcid.org/0000-0002-0930-4516 \\ Maria Paula Garrido Parraguez \\ Universidad Católica del Maule, Chile, Chile \\ paula.garrido-parraguez@gmail.com \\ Cristian Martinez Villacura \\ Universidad Católica del Maule, Chile, Chile \\ mvillacura@gmail.com
}

DOI: https://doi.org/10.35811/rea.v8i0.3

Recepción: 26 Julio 2019

Aprobación: 12 Septiembre 2019

\section{Resumen:}

Esta investigación corresponde a un estudio de caso cualitativo que tiene como objetivo analizar el alcance de un modelo pedagógico innovador, Flipped Classroom, y su conexión con la asignatura académica "Lenguaje y comunicación". Se decidió llevar a cabo este proceso de investigación dentro de una escuela chilena que ha implementado formalmente esta modalidad en su plan de estudios, mediante la aplicación de instrumentos que permitieron la recopilación de datos. Los datos se obtuvieron a través de entrevistas semiestructuradas y se clasificaron en categorías de análisis como currículum, innovación, metodología, rol docente y conexión del modelo de clase invertida con las familias y la organización interna de la escuela. Asimismo, a través de un cuestionario abierto, se analizó la aplicación de esta modalidad en la escuela, lo que permitió conocer la implementación en la asignatura de Lenguaje y Comunicación. Entre los principales hallazgos, podemos destacar la importancia del trabajo colaborativo y autónomo en la modalidad de aula invertida, la clara preferencia de los estudiantes por metodologías participativas como el método Flipped Classroom (por lo tanto, se considera una forma efectiva de aprender) y, finalmente, la transformación del paradigma educativo de toda la comunidad escolar, a pesar de las complejidades del modelo.

Palabras Clave: educación, sociedad, aprendizaje, Flipped Classroom, innovación.

\section{ABSTRACT:}

This research corresponds to a qualitative case study which aims to analyze the scope of an innovative pedagogical model, Flipped Classroom, and its connection with the scholar subject "Language and Communication" (Language and Communication). It was decided to carry out this process of investigation inside a Chilean school that have formally implemented this modality in their curriculum, by applying instruments that enabled data collection. Data were obtained through semi-structured interviews and were classified into categories of analysis such as curriculum, innovation, methodology, teaching role and connection of the inverted class model to families and the internal organization of the school. Likewise, through an open-ended questionnaire, the application of this modality in the school was analyzed, which allowed the implementation in the Language and Communication subject to be known. Among the main findings, we can highlight he importance of collaborative and autonomous work in the inverted classroom modality, the clear preference of students for participatory methodologies such as the Flipped Classroom method (therefore, it is considered an effective way to learn) and, finally, the transformation of the educational paradigm of the entire school community, despite the complexities of the model.

KEYWORDS: education, society, learning, Flipped Classroom, innovation. 


\section{INTRODUCCIÓN}

Los cambios sociales y el surgimiento de nuevas tecnologías en la era de la información han transformado indiscutiblemente los modos de vida de las personas, exigiendo una mayor comprensión del mundo. Se han generado nuevas concepciones sociales, cimentadas en el desarrollo del conocimiento, proceso del que Chile no queda exento. Por ello, reconocer la relevancia e influencia de la tecnología y el discurso proveniente de los medios, es clave para deducir que la educación es un factor preponderante, que permite desarrollar en las personas la capacidad de adquirir habilidades, transformar sus conocimientos, fomentar la innovación y aplicar los aprendizajes en la resolución de problemas. En este sentido, Gómez y Macedo (2010), señalan que "las TIC son la innovación educativa del momento y permiten enfrentar a los docentes y alumnos a cambios determinantes en el quehacer diario del aula y en el proceso de enseñanza-aprendizaje de los mismos" (p.211). De Fontcuberta y Guerrero (2007) sostienen que, en cuanto a la importancia de los medios de comunicación "es necesario aceptar como un hecho su considerable impacto y propagación, reconociendo que constituyen un elemento fundamental de la cultura en el mundo contemporáneo" (p.88). De este modo, en los sistemas educativos actuales, surge la imperiosa necesidad de conexión entre individuos y sus nuevas formas de relacionarse, a través de los modelos de ciertas conductas, la difusión de ideas y valores de una nueva sociedad.

La educación se define como un proceso continuo, que se realiza durante toda la vida, y en diferentes contextos. Precisamente, el sistema educativo debería velar porque el trabajo de la escuela no se limite solo a la entrega de información. Si bien, es importante que los estudiantes adquieran los contenidos de cada disciplina, es aún más importante que sepan buscar información, evaluar críticamente, producir nueva información, valorar distintos tipos de conocimiento, usar herramientas tecnológicas, desarrollar valores, utilizar el conocimiento para resolver problemas, y concretar proyectos individuales y colectivos, que respondan a las demandas de la sociedad en que viven y en la que vivirán. "El conocimiento surge cuando la persona es capaz de atribuir significado a la información. Precisamente, una de las tareas de la educación será transformar la información en conocimiento" (Tourón, Santiago y Díez, 2014, p.10). Desde esta perspectiva, se ha realizado esta investigación con el fin de lograr visualizar cómo la implementación del modelo pedagógico Flipped Classroom, en un establecimiento educacional chileno, ha permitido que los estudiantes y futuros ciudadanos desarrollen habilidades, destrezas y actitudes propias del socioconstructivismo, necesarias para la sociedad del siglo XXI.

Bergmaan y Sams (2014) definen la clase invertida como aquella que asegura que los estudiantes reciban una educación personalizada y ajustada a sus necesidades individuales. De este modo, el modelo está diseñado para cubrir los requerimientos de todos los estudiantes, aquellos que se esfuerzan por aprender y aquellos que aprenden de forma superficial, haciendo hincapié en la responsabilidad docente de encontrar una manera de llegar a los estudiantes con diferentes necesidades educativas. Precisamente, la metodología Flipped Classroom "ayuda a que los estudiantes aprendan mejor el contenido, como también les ayuda a convertirse en mejores estudiantes. No solamente para que tengan buenos puntajes en las pruebas, sino que para que aprendan a un nivel mucho más profundo" (Bergmaan y Sams, 2014, p.6).

Con este trabajo se pretende mostrar una alternativa innovadora, capaz de enmendar, en cierto sentido, la necesidad de una educación integral, dirigida a potenciar las diferentes capacidades de los alumnos. De esta forma, realizamos un acercamiento a un modelo que se caracteriza por trabajar de una forma distinta a la habitual, invirtiendo la estructura de la clase tradicional y generando materiales conceptuales que los alumnos puedan ver y revisar anticipadamente y de manera autónoma, para lograr un aprendizaje que se construye desde el conocimiento previo hasta lo colaborativo.

Finalmente, la importancia de este estudio radica en comprender la implementación y uso de este modelo pedagógico, como contribución al desarrollo de aprendizajes y de capacidades intelectuales, con el objetivo de formar y preparar al estudiante para enfrentar los desafíos de la sociedad actual. 


\section{De la enseñanza tradicional a un nuevo modelo pedagógico}

En la búsqueda de mejorar la calidad de la educación en los establecimientos del país, se hace necesario redireccionar el paradigma educativo hacia uno que potencie el desarrollo de habilidades como eje central de la formación y contribuya a disminuir las desigualdades del sistema que conduce, facilitando el acceso al conocimiento, a través, de variados recursos y experiencias de aprendizaje. Para alcanzar este anhelo prospectivo, es fundamental que el actual contexto educativo avance hacia una nueva enseñanza.

En la actualidad la función formadora de la escuela, en ciertos contextos, ha sido desplazada por los Medios Masivos de Comunicación (MMC) (González y Ramos, 2013), esto implica que la exclusividad de la escuela se ha debilitado y las fuentes de información se han ido ampliando y diversificando a gran velocidad, dando lugar a que cualquier información que antes era irrefutable, ahora pueda ser revisada y cuestionada. Frente a este escenario, las comunidades en el mundo, tanto internacionales como nacionales, se encuentran influenciadas por modelos sociales al servicio del mercado, basados en principios económicos, donde el rol de la escuela tradicional no fomenta la cultura crítica, por el contrario, limita el quehacer de la formación a crear sujetos competentes para el trabajo con una débil capacidad de crítica a la sociedad en la que viven. Por su parte, la escuela tiende a repetir modelos arcaicos, mantener un sistema clasista, que limita a las clases sociales más bajas, marginando su participación en la sociedad y coartando una serie de derechos desde temprana edad, al proporcionar solamente una enseñanza de conocimientos mínimos y no una de tipo integral.

Este modelo de enseñanza tradicional, que predomina aún en la mayoría de los establecimientos educativos de Chile, es el encargado de reproducir patrones conductuales y académicos, y de resguardar ciertos poderes políticos y económicos que desean mantener un status o resguardar ciertos esquemas de clase. Un esquema de clase, se refiere a "grupos que comparten recursos de mercado, determinantes de sus oportunidades de vida, doblemente entendidas como niveles de bienestar económico y patrones de movilidad social” (Torche, 2006, p. 39). De esta forma, las oportunidades de acceso y calidad de los estudiantes en los diferentes niveles de la formación educacional son determinantes en su futuro, debido a que gran parte de la estructura social del país aspira a cubrir carencias que solo por medio de la educación se pueden alcanzar.

Una de las necesidades de transformar el modelo tradicional de enseñanza está directamente relacionada con la nueva sociedad imperante, denominada "sociedad de la información y del conocimiento", que está presente en contextos tan importantes como la escuela. En este sentido, "En la sociedad actual parece no haber discusión, sobre todo por las cada vez más complejas y sofisticadas tecnologías empleadas como medios para la comunicación e información, en donde las nuevas generaciones participan de forma natural, sin obstáculos" (Colina, 2008, p.6), y las generaciones adultas intentan incorporarse sorteando distancias, obstáculos y aprendizajes, para lograr adecuarse a los cambios impuestos por dichos avances tecnológicos. Un reflejo de esto es que los estudiantes sean nativos digitales en la inmediatez de las comunicaciones, y los docentes, aquellos sujetos que han tenido que adaptarse y capacitarse en este nuevo mundo digitalizado. De este modo, se hace imprescindible considerar otras formas de cultura en el aula, ya que la enseñanza tradicional no está preparada para enfrentar las necesidades actuales del individuo y las nuevas fuentes de información, debido a que carece de estrategias pedagógicas que puedan entender tal cantidad de conocimiento solo a través de instrumentos limitados, basados en evaluaciones estandarizadas. Así mismo, el incremento en el acceso de los estudiantes a la información ha provocado un impacto importante en los contextos de aprendizaje, debido a que los individuos se comunican, relacionan y participan de nuevas formas. Existe un mayor nivel de conciencia, mayores exigencias y demandas con múltiples propuestas. En estas circunstancias, surge la necesidad de orientar la educación hacia un modelo que centre su objetivo en el aprendizaje y no en la mera enseñanza. La educación ha enfrentado un gran desafío social, en cuanto a su capacidad de adaptación a los cambios propios del desarrollo de la ciencia y la tecnología, "debido no solo a la complejidad de los procesos, sino a la forma como ellos intervienen en la orientación cognitiva y afectiva del colectivo, moldeando el pensamiento de las personas de acuerdo a cómo procesan y asimilan 
esa información" (Colina, 2008, p.6). Es por ello que comprender la importancia del fenómeno de la globalización, es esencial para entender las trasformaciones que está experimentando la sociedad, en que los MMC y la inmediatez de las comunicaciones reclaman un cambio en los modelos educativos presentes. Las instituciones educativas, encargadas de preparar a futuros ciudadanos y hacerlos parte de la sociedad, tienen la responsabilidad de modificar sus objetivos y desarrollar otras capacidades en los estudiantes, que estén enfocadas en mejorar continuamente las habilidades y competencias. El mayor acceso a distintas fuentes de conocimiento favorece la capacidad reflexiva e incrementa los cuestionamientos de los sujetos. Así mismo, el docente guía la construcción del conocimiento y participa en la formación de los futuros ciudadanos.

\subsection{Innovación Educativa}

En el ámbito educativo, la innovación se plantea como un reto difícil de abordar, debido a las prácticas de enseñanza arraigadas en las estructuras políticas y organizacionales, reticentes a la implementación de nuevos modelos con el fin de beneficiar los procesos pedagógicos. En base a las investigaciones de la Organización para la Cooperación y el Desarrollo Económico (OCDE), se reconocen tres dimensiones esenciales observables en los establecimientos, sobre las cuales se sustentaría el cambio: la innovación en el núcleo pedagógico estudiante-profesor, las estrategias de innovación institucional (procesos explícitos de mejoramiento, rediseño, evaluación y estudio de los aprendizajes, estrategias de liderazgo, etc.) y la creación de alianzas con otras instituciones, colegios, centros culturales, empresas, museos, entre otros (Fundación Chile, 2016).

En relación a lo establecido por OCDE y UNICEF (2016), si bien, no existe una investigación científica rigurosa sobre los beneficios del uso de la tecnología en el aprendizaje, las nuevas tendencias abogan por aprovechar su potencial en el desarrollo de los procesos educativos debido a sus múltiples ventajas. Por ejemplo, al utilizarse adecuadamente, la tecnología computacional permite desarrollar una enseñanza multimedia, además de un importante nivel de interactividad, creación y recopilación de información, que, bajo otras circunstancias, no serían posibles. Aquí también es preciso hacer una distinción entre perspectivas de aprendizaje, con tecnología que se enfoca en la misma tecnología y aquellas que se enmarcan en el estudiante. En el primer caso, la importancia se enfoca en el uso de la tecnología y en dar acceso a medios digitales de punta a los estudiantes y profesores, lo cual no tiene un fin pedagógico. El segundo, se centra en cómo aprenden las personas y la tecnología se utiliza como un recurso a disposición del aprendizaje humano. En este contexto, el uso adecuado de este medio debiera apuntar a cubrir necesidades de estudiantes y docentes.

Hacer del trabajo con tecnologías una situación de aprendizaje provechosa dependerá en gran medida de la comprensión que se tenga sobre la forma en que las personas aprenden con esta clase de recursos, cuestión que debe tener presente especialmente el profesor. Alejados o no de los distintos aspectos delimitados por la investigación, el escenario educativo internacional ha sido testigo de la implementación de un variado espectro de metodologías didácticas que se alejan de la instrucción tradicional y que apuntan tanto al trabajo en nuevos ambientes de aprendizaje, así como a propuestas próximas a las teorías socio-constructivistas. En este sentido, Ramírez (2012) indica que las necesidades de la sociedad actual reclaman nuevas formas de educación, donde las prácticas se dirijan al autoaprendizaje, a una manera adecuada de usar y manejar la información, y a tomar conciencia social para desarrollar el crecimiento colectivo.

En relación a nuevas formas de plantear el trabajo en aula, el concepto de aprendizaje tuvo un desarrollo importante. "Hoy, el concepto dominante es el socio-constructivista; en él se entiende que el aprendizaje es configurado en gran parte por el contexto en que está situado y es construido activamente, a través de la negociación social con otros" (OCDE, 2016, p.3). Bajo la implementación de estos principios, que buscan la generación de medios que promuevan el trabajo colaborativo, la valoración del entorno de los estudiantes y el fomento del aprendizaje auto-regulado y constructivo, el escenario educativo se ha transformado y 
diversificado en búsqueda de estrategias más efectivas. En este sentido, si bien los conceptos teóricos no sugieren fórmulas concretas de implementación, brindan la posibilidad de un uso flexible y creativo por parte de los docentes, tomando también en cuenta el hecho de que no todo el aprendizaje se genera dentro de la sala de clases, sino que frecuentemente ocurre de forma implícita en la vida diaria.

En cuanto a la realidad nacional, las disposiciones del Ministerio de Educación (2008) se han ido adaptando en las últimas décadas, enfocándose en acciones y competencias que apuntan a la generación de ambientes de aprendizaje como los antes descritos. En este sentido, el dominio A del Marco para la Buena Enseñanza (MBE) señala: en la preparación de la enseñanza, el docente, junto con poseer un acabado conocimiento y comprensión de las disciplinas que enseña, debe manejar "herramientas pedagógicas que faciliten una adecuada mediación entre los contenidos, los estudiantes y el respectivo contexto de aprendizaje" (MINEDUC, 2008, p.8). Así también, hace mención a la valoración del contexto natural del estudiante, argumentando que los profesores "requieren estar familiarizados con las características de desarrollo correspondientes a la edad de sus alumnos, sus particularidades culturales y sociales, sus experiencias y sus conocimientos, habilidades y competencias respecto a las disciplinas" (MINEDUC, 2008, p.9). Por otra parte, en su dominio B: Creación de un ambiente propicio para el aprendizaje, indica que el profesor debe presentar situaciones de aprendizaje desafiantes y apropiadas para sus alumnos, circunstancia que implica aspectos motivacionales de los estudiantes y la inclusión de nuevas estrategias. De esta forma, se recalca la importancia de la incorporación de tecnologías digitales, tanto en la formación del profesorado como en la preparación de recursos efectivos de aprendizaje.

\subsection{Habilidades y competencias para el siglo XXI}

Las continuas transformaciones de la sociedad han traído, junto al cambio de siglo, la creciente preocupación por generar un sistema educativo capaz de entregar una formación adecuada a las demandas de los tiempos actuales, donde la información excesiva, la multiculturalidad y otras modificaciones propias del contexto no debieran quedar fuera del aula.

En la actualidad, los individuos necesitan dominar las tecnologías y manejar la gran cantidad de información disponible, y enfrentar desafíos colectivos como la sostenibilidad ambiental y la equidad social, entre otros, de ahí la necesidad de definir las destrezas imprescindibles para enfrentar un escenario difícil y cambiante. En este sentido, cada competencia involucra demandas complejas que movilizan recursos psicosociales en un contexto particular.

Cada vez son más los educadores que buscan aportar soluciones para el logro de aprendizajes y de una formación más acertada para los estudiantes del siglo XXI (Fundación Chile, 2016). El desafío de la escuela para hacer frente a un mundo cambiante es prepararse para desarrollar habilidades cognitivas, sociales y emocionales para un futuro aún desconocido. Para esto existen dos aspectos a tener en cuenta, por un lado, se evidencia que los contenidos del currículum están quedando rápidamente obsoletos y por otro, sabiendo que existe una gran cantidad de información contenida en internet y en otros medios, no se debe centrar la atención solo en retener contenidos, sino en enseñar a utilizar dicha información.

De esta forma, las habilidades a desarrollar para el siglo XXI se enfocan en diversos ámbitos como creatividad, con la cual los estudiantes deben construir su propio conocimiento en conjunto con productos y procesos novedosos; pensamiento crítico, competencia en la que los estudiantes llevan a cabo un proceso disciplinado activo e intelectualmente hábil en el análisis, aplicación y evaluación de la información; metacognición, capacidad de reflexión de los alumnos sobre su propio aprendizaje; colaboración, capacidad de involucrarse y reconocer los roles de cada miembro del grupo, para lograr un trabajo cooperativo y eficaz; comunicación, uso adecuado de las habilidades comunicativas orales y escritas para distintos propósitos; alfabetización en información, competencia para la resolución de problemas informativos como definir, buscar, evaluar, sintetizar o crear un nuevo producto; alfabetización en tecnologías digitales de 
información, competencias para el desempeño en el uso de herramientas para crear, comunicarse y trabajar en contextos digitales; ciudadanía, la cual requiere que el alumno comprenda los valores democráticos que conlleva la convivencia en sociedad; vida y carrera, habilidades que permiten al estudiante adaptarse a los cambios y gestionar la forma de vida que quiere para su futuro (Fundación Chile, 2016). Trabajar todas estas competencias significa un gran cambio en la forma de abordar el aprendizaje, desde un paradigma individualista a uno que desarrolle la colaboración, el aprendizaje social, la creación, la comunicación, entre otras capacidades que potencian una formación integral actualizada.

Por otra parte, la OCDE (2003) también plantea tres amplias categorías de competencias, propuestas como esenciales para la vida de las personas y el buen funcionamiento de la sociedad: usar herramientas de manera interactiva, en cuanto a tecnología y lenguaje; interactuar en grupos heterogéneos; y actuar de forma autónoma.

\subsection{Competencia digital}

En los tiempos actuales, se puede constatar que la relación del ser humano con las tecnologías se ha convertido en un aspecto necesario y complejo. En el caso de las tecnologías de la información y la comunicación, históricamente han desempeñado un papel fundamental en la cultura nacional, en medios como la imprenta, el teléfono y la radio. Sin embargo, cuando estos recursos comienzan a ser utilizados habitualmente por un largo tiempo, se incorporan de manera natural y casi imperceptible en la vida de las personas, sin que se tome real conciencia de cómo su uso ha ido cambiando la cultura.

De esta forma, se desarrolla el proceso denominado alfabetización digital, que hace referencia a la adquisición de competencias en el uso de tecnologías. Dicho concepto es el más usado a nivel internacional, mientras que en Europa se utiliza el término competencia digital. Esta idea forma parte de las ocho competencias claves para el aprendizaje permanente y se define como el uso seguro y crítico de las tecnologías de la sociedad de la información para el trabajo, el ocio y la comunicación. "Se sustenta en las competencias básicas en materia de TIC: el uso de ordenadores para obtener, evaluar, almacenar, producir, presentar e intercambiar información, comunicarse y participar en redes de colaboración a través de internet" (EsteveMon, Gisbert y Lázaro, 2016, p.40).

De Fontcuberta y Guerrero (2011) enfatizan que "Chile tiene que entender la importancia de la alfabetización mediática digital”. Para ella, la educación respecto a los medios de comunicación es esencial, ya que, de esta manera, las personas sabrán utilizar de mejor forma los medios y así se podrá exigir una mayor calidad en los contenidos. En la formación de los futuros docentes es necesaria la incorporación de módulos basados en la comprensión y posterior enseñanza de los MMC (Espinoza Guzmán, 2017). El currículum escolar debiera fomentar estas habilidades de manera pertinente, para que los niños, ciudadanos, puedan desarrollar de mejor forma la competencia comunicativa. Además, para revertir esta situación, se deberán implementar políticas públicas, adoptando medidas en este sentido, ya que la libertad de expresión y el derecho a la información contribuyen a establecer y mantener la democracia.

\subsection{Flipped Classroom}

Siguiendo lo propuesto en los apartados anteriores, debemos consignar y reafirmar la idea de la fuerte influencia de la tecnología y MMC en las nuevas formas de aprendizaje, propias de una sociedad globalizada y cambiante, con sistemas educativos que comienzan a incorporar innovaciones en aula, y donde el universo de información existente se vuelve cada vez más accesible. La irrupción del modelo pedagógico Flipedd Classroom surge de la combinación de distintas metodologías constructivistas, aprovechando los recursos digitales disponibles. Según Bergmann y Sams (2014), en la denominada clase invertida, los acontecimientos 
que comúnmente ocurren dentro de la escuela, se llevan a cabo ahora fuera de ella. De esta forma, el material digital creado por los docentes es visualizado por los estudiantes antes de la clase, permitiendo hacer del aula un espacio para resolver problemas y trabajar de forma colaborativa.

El término Flipped Classroom fue acuñado por los profesores de química Jonathan Bergmann y Aaron Sams, en Woodland Park, Colorado, Estados Unidos. Los docentes, desarrollaron una iniciativa para ayudar a aquellos estudiantes que perdían clases por distintas razones, entre ellas, las asociadas a las extensas distancias de la zona semirural a la que pertenecían. Bergmann y Sams (2014) comenzaron en el año 2007 a grabar sus propias clases, usando un software de grabación de imágenes, las que subieron a internet para que sus estudiantes tuvieran acceso. Esto les permitió darse cuenta de que la nueva modalidad posibilitaba centrarse en las necesidades de aprendizaje individuales de los estudiantes, y no perdían tanto tiempo en re-enseñar los contenidos perdidos por los alumnos que no asistían al colegio. De este modo, el modelo Flipped Classroom "habla el idioma de los estudiantes de hoy, quienes están acostumbrados a recurrir a la web y a las redes sociales para obtener información e interacción” (Tourón y Santiago, 2015, p.222). En primer lugar, el tiempo ideal para cada video, sugerido por los autores, es de cinco minutos de duración, ya que, "algunas investigaciones muestran que la novedad de cualquier estímulo tiende a desaparecer después de unos 10 minutos, y como resultado, los alumnos demandan nuevos contenidos después de ese corto espacio de tiempo" (Tourón y Santiago, 2015, p.222). En segundo lugar, se encuentra la clase propiamente tal, diseñada como un taller donde el estudiante puede resolver las dudas generadas durante la visualización del recurso, permitiendo, principalmente, un apoyo un tanto más personalizado por parte del docente.

El desarrollo del modelo pedagógico de aula invertida, debido a la versatilidad de sus características y su naturaleza esencialmente práctica, basado en el rol activo del estudiante, se relaciona con una serie de constructos como:

a. El Socioconstructivismo: basado en las investigaciones de autores como Piaget, Ausubel, Bruner y Vigotski, propone una explicación a la naturaleza del conocimiento humano, enfocada en el aprendizaje activo y social, elementos esenciales de la educación actual, y, por ende, de las innovaciones didácticas. El constructivismo se centra en el estudiante, quien genera procesos de producción de sentido a su aprendizaje, y el socioconstructivismo, en la interrelación del alumno y su cultura, quien establece aprendizajes como consecuencia de su desarrollo y la relación con los demás.

b. Aprendizaje cooperativo y colaborativo: algunos autores hacen distinción entre aprendizaje cooperativo y colaborativo, ambos modelos sitúan al alumno como eje central de su aprendizaje, aunque no como exclusivo responsable de este. Según Zañartu (2003), ambos se diferencian en que mientras el primero necesita de una estructuración mayor de parte del docente para realizar una actividad, el segundo requiere mayor autonomía del grupo de estudiantes y menos estructuración por parte del profesor.

c. Aprendizaje entre pares: como expresa Pinargote (2014), el método de enseñanza interactivo "instrucción entre pares" nació de los cuestionamientos de su autor, Eric Mazur, sobre el tradicional método de enseñanza tipo conferencia y su forma de presentación del material de estudio. Frecuentemente, esta información proviene directamente de los textos guías o de las notas de lectura creadas por el profesor, lo que da lugar a una audiencia pasiva y muy poco incentivada de atender la clase.

d. Aprendizajes basados en indagación: es un enfoque enmarcado en la participación activa, orientado a que los estudiantes piensen en forma sistemática o investiguen para llegar a soluciones razonables a un problema. Este tipo de enseñanza se centra en el alumno, no en el docente y promueve el trabajo intelectual entre estudiantes, basado en problemas, no en soluciones.

e. Neuroeducación: para lograr un aprendizaje óptimo, aún en situaciones ideales, es preciso conocer de qué manera funciona el cerebro y, por ende, cómo aprende el ser humano, cuestionamiento muchas veces olvidado en el desarrollo de estrategias pedagógicas. Es precisamente, a través de la Neurociencia, que se intenta dar respuesta a esta interrogante. Se debe reconocer que el estudio del cerebro es un proceso bastante complejo, pero gracias a los datos aportados por la neurociencia, se dispone de información empírica que 
permite comprender y poner en práctica distintos programas y metodologías educativas innovadoras, afines a las competencias, necesidades personales y de aprendizaje que presentan los alumnos.

Son variados los aspectos que se relacionan con un proceso de innovación educativa como Flipped Classroom, que propone un cambio sustancial en el papel del docente, la escuela y el alumno, para dar cabida a una forma nueva de concebir el aprendizaje. Se requiere cambiar el espacio del aula a un lugar para la cooperación entre alumnos, ir más allá de la mecanización y repetición de los deberes y sustituirlos por tareas que supongan un reto para los estudiantes. Todo esto, con el objetivo de preparar a las nuevas generaciones para un mundo cambiante y ofrecerles los recursos necesarios para adaptarse a una sociedad versátil, que exige aprender a aprender.

\section{Método De INVEstigación}

La investigación, mediante el uso de una metodología cualitativa, tiene como propósito analizar los alcances del modelo Flipped Classroom en un contexto educacional chileno, con enfoque en la asignatura de Lenguaje y Comunicación. Mientras que los objetivos específicos a los que busca responder son:

1. Conocer la implementación del modelo Flipped Classroom en un establecimiento educacional chileno.

2. Comprender el modelo Flipped Classroom desde la asignatura de Lenguaje y Comunicación.

Para dar cumplimiento a los objetivos anteriores, se implementó un cuestionario y focus group, aplicado a directivos, profesores y alumnos.

Esta investigación se basa en una acabada descripción contextual de los hechos (Bisquerra, 2003), como un reflejo de la realidad observada en el Colegio Mayor de Tobalaba, por medio de una recolección de datos que permitió el análisis e interpretación del fenómeno que fue observado. En este sentido, la metodología cualitativa se refiere a "la investigación que produce datos descriptivos: las propias palabras de las personas, habladas o escritas, y la conducta observable" (Taylor y Bogdan, 1987, p.20). De esta forma, los sucesos se analizaron desde la perspectiva de los participantes de un contexto particular, específicamente, los distintos actores de un establecimiento educacional que ha adoptado a nivel institucional, un modelo pedagógico innovador como el Flipped Classroom. Por lo tanto, la presente investigación, de acuerdo a Hernández, Fernández y Baptista (2006), involucró la recolección de datos utilizando técnicas que no pretenden medir ni asociar los resultados con números. Por el contario, se refieren a instrumentos tales como observación no estructurada, entrevistas abiertas, revisión de documentos, discusión en grupo, evaluación de experiencias personales e interacción con una comunidad en particular.

\subsection{Población}

Bisquerra (2003) describe población como un "conjunto de todos los individuos (objetos, personas, eventos) en los que se debe estudiar un fenómeno” (p.143). En relación a este criterio, la investigación contempló dos establecimientos educacionales chilenos que utilizan el modelo Flipped Classroom a nivel institucional, de los cuales, se optó por uno en particular, pues demostró interés y disponibilidad con la investigación, específicamente, el Colegio Mayor, sede Tobalaba.

\subsection{Muestra}

Según Hernández, Fernández y Baptista (2006), muestra se define como "un subconjunto de la población, una unidad de análisis o grupo de personas, contextos, eventos o sucesos, sobre la cual se recolectan los datos, sin que necesariamente sean representativos del universo" (p.173). Conforme a lo mencionado anteriormente, el grupo de personas escogido en esta selección involucró los niveles: institucionales, 
curricular y de aula, contemplando de esta manera, a directivos (directores y UTP), profesores (Lenguaje y Comunicación) y estudiantes (EB y EM) del Colegio Mayor Tobalaba, según la disponibilidad del establecimiento. En los niveles directivos, profesores y estudiantes, se aplicaron cuestionarios, entrevistas y focus group que permitieron responder a uno de los objetivos de la investigación.

El periodo de recolección de cada uno de estos datos, fue durante tres semanas, mediante reuniones de dos horas, dos días a la semana y en horarios acordados con cada uno de los grupos mencionados anteriormente.

\section{Resultados}

Con el fin de extraer mayor información acerca de la implementación del modelo de aula invertida en un contexto educacional chileno, se realizaron y analizaron las entrevistas semiestructuradas a diferentes profesionales, pertenecientes a los distintos niveles organizacionales del Colegio Mayor Tobalaba. Para ello, se confeccionó una matriz de análisis, determinando en base a la evidencia empírica, categorías y subcategorías para una mejor comprensión de la información, de acuerdo a lo expuesto en la tabla 1.

TABLA 1

Categorías y subcategorías

\begin{tabular}{|l|l|}
\hline CATEGORÍA & SUBCATEGORİA \\
\hline \multirow{2}{*}{ CURRICULO } & Planes y Programas \\
\cline { 2 - 2 } & Adecuaciones Curriculares \\
\hline \multirow{4}{*}{ METODOVACIÓN } & Implementación \\
\cline { 2 - 2 } & Capacitación docente \\
\cline { 2 - 2 } & $\begin{array}{l}\text { Planificación } \\
\text { apresos de enseñanza - }\end{array}$ \\
\cline { 2 - 2 } & Recursos didácticos (TICS) \\
\cline { 2 - 2 } & Evaluación \\
\cline { 2 - 2 } & Asignatura y Aula invertida \\
\hline ROL DOCENTE & Relación docente alumno \\
\hline RELACIÓN CON LA FAMILIA & \\
\hline $\begin{array}{l}\text { ORGANIZACIÓN } \\
\text { INSTITUCIONAL }\end{array}$ & \\
\hline
\end{tabular}

Elaboración propia

De acuerdo al análisis general de las entrevistas semiestructuradas, se presentaron seis segmentos. Entre estos, se encuentra la categoría currículum, conformada por la subcategoría planes y programas, donde se han realizado modificaciones sustanciales en el nivel de educación parvularia, no así de primero básico a cuarto medio, cursos en los que se continúa utilizando el modelo propuesto por el Ministerio de Educación (2011); y la subcategoría adecuaciones curriculares, que demostró la utilización de un $20 \%$ de clases bajo la modalidad de aula invertida, incluyendo trabajo por proyectos, aumento en la cantidad de horas de inglés y mayor espacio para los docentes, en la planificación y confección de material digital.

1. La categoría innovación, comprende la subcategoría implementación, relacionada principalmente con la necesidad de equiparar tecnológicamente el establecimiento, de acuerdo a su proyecto de innovación, lo que implicó instalar infraestructura adecuada, como: estudio de grabación, cabinas de edición, plataforma virtual y servidor de video, entre otros, y, por ende, significó una importante inversión para la comunidad educativa. En esta categoría, es relevante comprender que la implementación tecnológica, va de la mano con la capacitación docente, para desarrollar de manera eficiente y eficaz este modelo pedagógico.

2. La categoría metodología, que comprende las subcategorías: planificación, procesos de enseñanza - aprendizaje, TICS, evaluación y asignatura y aula invertida, nos permite señalar que el proceso de 
planificación (anual y bimensual) es importante para potenciar la formación de personas autónomas, a través de clases dinámicas y colaborativas, que contemplan el uso de cápsulas de conocimiento en distintos formatos, todo ello en coherencia con menos instancias de evaluación sumativas y más instancias formativas, donde se incluyen también reflexiones sobre la práctica pedagógica.

3. La categoría rol docente logra evidenciar la importancia del educador como agente de cambio cultural, lo que se relaciona con la motivación, autonomía y perfeccionamiento permanente, así como con el trabajo colaborativo con los pares, donde también se generan reticencias por la exposición que muchas de estas nuevas prácticas conllevan. La subcategoría relación docente-alumno destaca principalmente el cambio de roles en el modelo de aula invertida, donde el estudiante cobra un rol protagonista.

4. La categoría relación con las familias evidencia que la participación del entorno cercano del alumno en el logro de aprendizajes significativos es fundamental, especialmente en el cierre de unidades, desarrollo de proyectos de fomento lector, y en asignaturas con clase invertida como: Lenguaje y Comunicación, Historia, y Matemáticas, donde el rol otorgado tradicionalmente a la familia también tiende a cambiar.

5. La categoría organización institucional interna del establecimiento muestra algunos roles y cambios en la planta profesional y los equipos participantes, como, por ejemplo, la modificación de la función de directores de ciclo, desde un rol administrativo a un contexto curricular, y la importancia del Centro de Información y Gestión de Aprendizajes como organismo asesor, que orienta la implementación de las clases invertidas. Finalmente, en las impresiones de los distintos actores involucrados destaca la importancia del factor humano, incluso por sobre la implementación tecnológica y la utilización de recursos digitales.

En el análisis del cuestionario de preguntas abiertas, que fue respondido vía digital, se utilizó un formato que permitió ordenar la información. Luego, se clasificaron como categorías de análisis, las cuales representaron los aspectos esenciales en el aula. Entre los aspectos fundamentales del instrumento, se consideraron la experiencia del docente, el diseño de la planificación, los recursos y actividades, la evaluación y los tres principales ejes del área de Lenguaje y Comunicación, entre otros elementos que se materializan en el desarrollo de una clase. Posteriormente, se elaboró una matriz de análisis, donde se ordenó la evidencia empírica recolectada y se realizaron interpretaciones preliminares de cada una de las categorías. En el análisis general del cuestionario de preguntas abiertas, se presentaron doce segmentos. Entre estos, se encuentra la categoría aula invertida en la asignatura de Lenguaje y Comunicación.

6. La categoría aula invertida demostró alcances positivos en términos de innovación, pues otorgó a los docentes mayor libertad en la utilización de diversos recursos tecnológicos y posibilitó un desarrollo de contenidos claro y didáctico, que permite a los estudiantes empoderarse de su aprendizaje y mejorar sus prácticas en cuanto a trabajo en equipo. La adaptación a este cambio implicó, por ejemplo, disposición y tiempo para preparar el material, lo que permitió demostrar que una clase taller requiere de una labor profunda. De esta forma, el trabajo en conjunto con otros docentes, se encuentra en permanente reflexión.

7. La categoría planificación bajo el modelo de aula invertida incluye que los docentes lleven a cabo un trabajo en conjunto de dichas unidades integradas, para enriquecer los aprendizajes. La categoría de estrategias y recursos utilizados para preparar una cápsula de conocimiento, hace mención al empleo de variados recursos, desde presentaciones PowerPoint o Prezi, hasta el uso de hipervínculos con videos apropiados al contenido, los temas más complejos poseen mayor soporte audiovisual.

8. La categoría desarrollo de una clase taller, según el modelo Flipped Classroom, contempla ciertas etapas, como selección del contenido, creación de un guion o búsqueda de material que sirva de desencadenante del aprendizaje, seguidas del proceso de edición, con el apoyo del departamento de tecnología y aprendizaje, para dar forma a un material adecuado al taller. Una parte esencial, luego que el recurso ha sido visto, se relaciona con la acción de los profesores, retroalimentando y orientando la tarea práctica, privilegiando la creación y el uso del conocimiento personal del estudiante. 
9. La categoría enfocada en el eje escritura, a través del modelo Flipped Classroom, se potencia a través del trabajo metódico y ordenado en la producción de textos, ya sea, en la creación o transformación de un escrito, permitiendo identificar a los alumnos que presentan mayores dificultades y mejorando habilidades del proceso de escritura. En la categoría desarrollo del eje lectura, los docentes manifiestan trabajar en menor medida en esta área, ya que, los estudiantes demuestran ser buenos lectores. Podemos concluir, que el eje escritura es el que más se beneficia con el modelo de clase invertida, en la asignatura de Lenguaje y Comunicación.

10. La categoría evaluación del aprendizaje, a través del modelo de aula invertida, evidencia que los docentes evalúan, a través de rúbricas, pautas de cotejo y/o de coevaluación, ajustadas a dinámicas de trabajos grupales y luego retroalimentan, para que los alumnos tengan la oportunidad de verificar y corregir su trabajo. La categoría ventajas y desventajas de la implementación del modelo Flipped Classroom en la asignatura de Lenguaje y Comunicación ofrece aspectos positivos, como la variedad de formas con que se puede abordar un contenido, lo que posibilita que la clase sea un espacio donde el estudiante sea protagonista de su propio aprendizaje, con contenidos recuperables fácilmente.

Un aspecto negativo surge cuando el alumno no observa la cápsula de aprendizaje, ya que se retrasa el proceso de continuidad y ejecución del contenido. La categoría asociada a cuál modelo, invertido o tradicional, entrega mayores beneficios a la asignatura de Lenguaje y Comunicación, refleja un acuerdo entre los educadores, manifestando una visión positiva en cuanto a la modalidad investigada, ya que, se desarrollarían mejores habilidades, por ejemplo, a través del trabajo por proyectos.

Finalmente, según los docentes, el modelo pedagógico Flipped Classroom, que aún experimenta innumerables transformaciones en el establecimiento, ha permitido cambiar progresivamente el paradigma educativo tradicional, por uno centrado en el aprendizaje del alumno y sus intereses, con mayor flexibilidad en la aplicación de estrategias pedagógicas y que se desarrolla a la par con las comunicaciones y la tecnología, no obstante, este cambio implica una serie de elementos fundamentales para su implementación, entre los que se encuentran el tiempo de preparación de las cápsulas de contenido y la poca seguridad de su visualización por parte de los estudiantes.

\section{Otros hallazgos de LA INVESTigación}

Creemos relevante referirnos a tres hallazgos significativos que se desprenden de esta investigación y que están relacionados con el trabajo colaborativo, la preferencia de los estudiantes por metodologías novedosas y que fomentan el pensamiento crítico y la transformación del paradigma educativo.

El primer hallazgo, hace referencia a Platero, Tejeiro, y Reis (2015) quienes plantean los beneficios del trabajo colaborativo en ciertas temáticas, durante la implementación de un proyecto académico. Esto coincide en gran medida con las experiencias de clase del Colegio Mayor Tobalaba, donde el trabajo colectivo y autónomo propio de la modalidad de aula invertida favorece el desarrollo de ciertas asignaturas, en especial las humanistas, aportando un significativo factor de motivación en los estudiantes debido, precisamente, a las características de este tipo de actividades, las que apuntan principalmente a la creación grupal, por medio de estrategias basadas en la asignación definida de roles.

Otra idea importante de destacar tiene relación con la percepción de los alumnos en cuanto a este tipo de nuevas metodologías: fue posible determinar que la implementación del modelo Flipped Classroom es altamente valorada, pues el foco está puesto en el desarrollo integral de la persona y no en la mecanización ni estandarización del aprendizaje.

Por último, uno de los hallazgos relevantes de la investigación se centra en la transformación del paradigma educativo. Fue posible determinar que para la adecuada implementación del modelo Flipped Classroom, 
fue necesaria una serie de cambios, entre ellos el factor humano, al que se suman los ámbitos estructurales, aspectos curriculares del colegio y, en definitiva, el involucramiento de toda la comunidad educativa.

Finalmente, cabe señalar que el Colegio Mayor continúa funcionando con planes y programas propuestos por el Ministerio de Educación, con ciertas adaptaciones de acuerdo a las exigencias que se plantean desde su PEI, pero se ve limitado en aspectos metodológicos, por lo que se proyecta la necesidad de implementar un currículum propio a corto plazo.

\section{Conclusiones}

La educación es un proceso cultural en constante evolución, que se modifica a la par con las transformaciones sociales y el desarrollo de competencias individuales que estas demandan. Frente a estos cambios, en el ámbito nacional, surge la necesidad de generar nuevos métodos de enseñanza y aprendizaje, acordes con una sociedad globalizada y dinámica, donde el uso masivo de tecnologías en distintas áreas se hace indispensable. De esta manera, en un contexto dominado por los MMC y las tecnologías, nace la oportunidad de reformular la enseñanza tradicional, poniendo nuevos elementos al servicio del aprendizaje de los estudiantes. Bajo este objetivo, se implementan métodos innovadores como el modelo Flipped Classroom, o clase invertida, que traslada el foco de atención al estudiante, modificando el rol pasivo que le otorga la enseñanza clásica.

Para dar respuesta a los objetivos de esta investigación, se crearon preguntas enfocadas en conocer la implementación del modelo en un establecimiento educacional chileno y comprender su aplicación desde la asignatura de Lenguaje y Comunicación. De esta forma, bajo la primera interrogante, se profundizaron conocimientos con respecto a la concreción del modelo de aula invertida en el establecimiento investigado, a través de entrevistas semiestructuradas. Frente a la necesidad de clasificar la información recopilada para su posterior interpretación, se generaron seis grandes categorías: currículum, innovación, metodología, rol docente, relación familia y aula invertida, y organización interna. Algunos de estos ámbitos, se dividieron en subcategorías como: planes y programas, adecuaciones curriculares, implementación, capacitación docente, planificación, procesos de enseñanza y aprendizaje, recursos didácticos, evaluación, asignatura y aula invertida, y relación docente con el alumno. Este método de investigación nos llevó a concluir que el modelo pedagógico Flipped Classroom, no tiene márgenes rígidos en su aplicación, por lo que el formato adoptado por el Colegio Mayor Tobalaba, corresponde a una configuración acorde a intereses internos, en este caso, incluyendo una gran inversión en equipamiento y una distribución de cargos coherente con el manejo de dicho formato. En relación a esto, si bien se ha puesto énfasis en la implementación tecnológica, el principal pilar sobre el cual se sustenta el modelo, es el recurso humano. Aquí se presenta, por ejemplo, un importante cambio en el rol del profesor, no solo en el foco de la enseñanza, sino también en su reflexión profesional, planteándose como un docente en formación constante y con competencias digitales, capaz de cuestionar sus falencias y proponer nuevos recursos que contribuyan a mejorar las prácticas educativas. Desde el factor humano también se identifican las principales reticencias al modelo de aula invertida: en los profesores, el recelo a la modernización y a la exposición, ya que implica la evaluación constante del trabajo docente; en los estudiantes, la resistencia por haber comenzado su formación bajo el modelo expositivo de clases.

En relación al segundo cuestionamiento de la investigación, se interpretó la información entregada por los cuestionarios de preguntas abiertas, realizados a los docentes del área de Lenguaje y Comunicación, con el fin de comprender la aplicación de la modalidad investigada desde dicha asignatura. En este asunto, determinamos que el modelo es valorado positivamente por los docentes del área de Lenguaje y Comunicación, quienes destacan más virtudes que defectos, principalmente en el eje de escritura.

Finalmente, a pesar de las complejidades propias de la implementación de un nuevo modelo pedagógico en el contexto educativo nacional, es posible identificar una visión positiva de la incorporación de Flipped Classroom en el Colegio Mayor Tobalaba, el que ha logrado generar un diseño estratégico de una modalidad innovadora, hasta insertarla a nivel institucional, teniendo en cuenta el tiempo y los procesos de adaptación 
que se requieren, con la finalidad de encontrar nuevas herramientas que permitan mejorar el trabajo docente, y otorgar posibilidades de aprendizaje y desarrollo a los estudiantes del siglo XXI.

\section{ReFERENCIAS}

Bergmann, J. y Sams, A. (2014). Dale la vuelta a tu clase. Lleva tu clase a cada estudiante, en cualquier momento y cualquier lugar. Madrid: SM.

Bisquerra, R. (2003). Metodología de la investigación educativa. Madrid: Editorial La Muralla S.A.

Colina, L. (2008). Las Tics en el proceso de enseñanza-aprendizaje en la educación a distancia. Revista de Educación Laurus, 28, 295- 313.

de FONTCUBERTA, M., \& Guerrero, C. (2011). Una nueva propuesta para la educación en medios / A New Proposal for Media Education. Cuadernos.Info, (20), 87-97. https://doi.org/10.7764/cdi.20.115.

Espinoza Guzmán, A. (2017). La enseñanza de la publicidad en la escuela chilena: debilidades y desafíos. Revista de Educación de la Universidad de Granada, 24, 185-202.

Esteve-Mon, F., Gisbert, M. y Lázaro, J. (2016). La competencia digital de los futuros docentes: ¿cómo se ven los actuales estudiantes de educación? Perspectiva educacional, 55(2), 38-54.

Fundación Chile (2016). Habilidades para el siglo XXI. Innovación Educativa (N³)

Gómez, L. y Macedo, J. (2010). Importancia de las Tic en la educación básica regular. Investigación Educativa, 14(25), 209-224.

González, R. y Ramos, P. (2013). La Otra Educación: red de escuelas libres en Chile. Tesis para optar al título de educadora de párvulos y educación básica Inicial. Universidad de Chile, Chile.

Hernández, R. Fernández, C. y Baptista, P. (2006). Metodología de la investigación (quinta edición). México D.F: Editorial Mc Graw Hill.

Ministerio de Educación de Chile (2008). Marco para la Buena Enseñanza. Ministerio de Educación, Chile.

Ministerio de Educación de Chile (2011). Bases Curriculares. Ministerio de Educación, Chile.

Organización para la Cooperación y el Desarrollo Económico (2003). La definición y selección de competencias clave. Resumen Ejecutivo. OCDE.

Organización para la Cooperación y el Desarrollo Económico y Fondo de las Naciones Unidas para la Infancia (2016). La Naturaleza del Aprendizaje: Usando la investigación para inspirar la práctica. Guia del practicante. Informe OECD.

Organización para la Cooperación y el Desarrollo Económico y Fondo de las Naciones Unidas para la Infancia (2016). La Naturaleza del Aprendizaje: usando la investigación para inspirar la práctica. Informe OCDE.

Pinargote, K. (2014). Instrucción entre pares, un método sencillo pero efectivo para enseñar. Revista Fenopina, 4(7), 56-59.

Platero, M., Tejeiro, M., y Reis, F. (2015). La aplicación de Flipped Classroom en el curso de dirección estratégica. Tesis de la Universidad Europea de Madrid, España.

Ramírez, M. (2012). Modelos y estrategias de enseñanza para ambientes innovadores. México: Editorial Digital Tecnológico de Monterrey.

Taylor, S. y Bogdan, R. (1987). Introducción a los métodos cualitativos de investigación. México: Editorial Paidós.

Torche, F. (2006). Una clasificación de clases para la sociedad chilena. Revista de sociología, 20, 15-43. https:// doi.org/10.5354/0719-529X.2006.27529

Tourón, J.; Santiago, R. y Díez, A. (2014). The Flipped Classroom. Cómo convertir la escuela en un espacio de aprendizaje. España: Editorial Grupo Océano.

Tourón, J. y Santiago, R. (2015). El modelo Flipped Learning y el desarrollo del talento en la escuela. Revista de Educación, 368, 196-231. 
Alejandro Espinoza Guzmán, et al. El modelo pedagógico Flipped Classroom: contribución al desarkol...

Zañartu, L. (2003). Aprendizaje colaborativo: una nueva forma de diálogo Interpersonal y en Red. Contexto Educativo, Revista digital de Educación y Nuevas Tecnologías, 28.

\section{BY-ND}

